ESAIM: M2AN

Vol. 40, No 2, 2006, pp. 413-430

DOI: $10.1051 / \mathrm{m} 2 \mathrm{an}: 2006017$
ESAIM: Mathematical Modelling and Numerical Analysis

www.edpsciences.org/m2an

\title{
DISCRETE COMPACTNESS FOR A DISCONTINUOUS GALERKIN APPROXIMATION OF MAXWELL'S SYSTEM
}

\author{
Emmanuel Creusé ${ }^{1}$ And Serge Nicaise ${ }^{1}$
}

\begin{abstract}
In this paper we prove the discrete compactness property for a discontinuous Galerkin approximation of Maxwell's system on quite general tetrahedral meshes. As a consequence, a discrete Friedrichs inequality is obtained and the convergence of the discrete eigenvalues to the continuous ones is deduced using the theory of collectively compact operators. Some numerical experiments confirm the theoretical predictions.
\end{abstract}

Mathematics Subject Classification. 65N25, 65N30.

Received: July 11, 2005.

\section{INTRODUCTION}

In this paper we deal with the discrete compactness property for a discontinuous Galerkin approximation of Maxwell's system on quite general tetrahedral meshes. This property has been studied for standard Galerkin approximation for a quite large family of edge elements on two and three dimensional domains $[7,8,15,21,26]$. But to our knowledge this property is not yet proved for the discontinuous Galerkin method. We here concentrate on the interior penalty method introduced in [19] (see also [24] for two-dimensional domains).

The success of DG methods is today well recognized and is mainly due to its flexibility in the choice of the approximation space, since it allows the use of meshes with hanging nodes and local spaces of different orders. This renders this method well suited for $h-p$ adaptivity. Furthermore the implementation of DG methods is quite easy by using elementwise shape functions, a great advantage for elements of high order, such a property is not satisfied for standard edge elements.

Our proof of the discrete compactness property is based on the same property for the standard Galerkin approximation proved in [26] and the use of a decomposition of the discontinuous approximation space into a continuous one and its orthogonal for an appropriate inner product similar to [19,20] (but different from the one used in these references). The discrete Friedrichs inequality follows from this discrete compactness property and a contradiction argument. The convergence of the discrete eigenvalues to the continuous ones is deduced using the theory of collectively compact operators [1], which requires pointwise convergence of the sequence of the discrete operators (see [26] for the use of this approach for the standard Galerkin approximation of Maxwell's system). In our case, the collectively compact property is deduced from the discrete compactness property and

Keywords and phrases. DG method, Maxwell's system, discrete compactness, eigenvalue approximation.

1 Université de Valenciennes et du Hainaut Cambrésis, MACS, Institut des Sciences et Techniques de Valenciennes,

59313 Valenciennes Cedex 9, France. Emmanuel.Creuse@univ-valenciennes.fr; Serge.Nicaise@univ-valenciennes.fr

(C) EDP Sciences, SMAI 2006 
the pointwise convergence is obtained by introducing mixed formulations and using a variant of the second Strang lemma.

Let us notice that we restrict ourselves to the $h$-version of the method, even if we consider a discontinuous Galerkin method for polynomials of order less than $k$, for any positive integer $k$, we do not estimate the dependence of the constant with respect to $k$. The extension of our analysis to the so-called $h-p$ version needs more investigations (see [9] for rectangular meshes). The convergence analysis of the DG method for the source problem by using the results of this paper also requires some further analysis.

For the numerical experiments, since the null space of the operator is relatively large, we have used a discrete regularization method that allows us to work in the setting of positive definite matrices $($ see $[3,18]$ for the standard edge elements).

The paper is organized as follows. Section 2 introduces the problem and some notation. The discretization and the discontinuous Galerkin method are given in Section 3. Section 4 contains the main results of the paper, namely the proof of the discrete compactness property and of the discrete Friedrichs inequality. The discrete eigenvalue problem is presented in Section 5 . In Section 6, we first recall some results about collectively compact operators and then used them to deduce the convergence of the eigenvalues. Finally some numerical experiments are presented in Section 7, that confirm the theoretical predictions. The obtained numerical eigenvalues are furthermore comparable with the ones given in the existing literature.

\section{Preliminaries AND NOtation}

Let us fix a bounded domain $\Omega$ of $\mathbb{R}^{3}$ with a Lipschitz polyhedral boundary $\Gamma$. We denote by $\boldsymbol{n}$ the unit outward normal vector along $\Gamma$. For the sake of simplicity we assume that $\Gamma$ is simply connected.

On this domain we consider the following eigenvalue problem: Find an electric field $\boldsymbol{E} \neq 0$ and an electric eigenvalue $\lambda$ such that

$$
\begin{array}{r}
\text { curl curl } \boldsymbol{E}=\lambda \boldsymbol{E} \text { in } \Omega, \\
\operatorname{div} \boldsymbol{E}=0 \text { in } \Omega, \\
\boldsymbol{E} \times \boldsymbol{n}=0 \text { on } \Gamma .
\end{array}
$$

This eigenvalue problem has been studied extensively in the literature, see e.g. [23,25]. It is well known that under the above assumptions, the electric eigenvalues are real and positive and that the set of eigenvalues is discrete. Moreover if $\lambda$ is an electric eigenvalue, then the eigenspace is finite dimensional.

For further purposes, we recall the variational formulation of this problem. It requires the use of the following (real) function spaces:

$$
\begin{aligned}
\mathcal{H}_{0}(\operatorname{curl} ; \Omega) & =\left\{\boldsymbol{u} \in L^{2}(\Omega)^{3}: \operatorname{curl} \boldsymbol{u} \in L^{2}(\Omega)^{3} \text { and } \boldsymbol{u} \times \boldsymbol{n}=0 \text { on } \Gamma\right\}, \\
X & =\left\{\boldsymbol{u} \in \mathcal{H}_{0}(\operatorname{curl} ; \Omega): \operatorname{div} \boldsymbol{u}=0 \text { in } \Omega\right\}
\end{aligned}
$$

both spaces being equipped with the norm

$$
\|\boldsymbol{u}\|_{\mathcal{H}_{0}(\operatorname{curl} ; \Omega)}=\left(\|\boldsymbol{u}\|^{2}+\|\operatorname{curl} \boldsymbol{u}\|^{2}\right)^{1 / 2}
$$

where here and below $\|\cdot\|$ means the $L^{2}(\Omega)^{3}$ (or $L^{2}(\Omega)$ according to the context) norm. Furthermore for any $\boldsymbol{u}, \boldsymbol{v} \in L^{2}(\Omega)^{3}$, we will denote

$$
(\boldsymbol{u}, \boldsymbol{v})=\int_{\Omega} \boldsymbol{u} \cdot \boldsymbol{v}
$$

their standard inner product.

In view of (2), the weak formulation of the electric eigenvalue problem is to find $\boldsymbol{E} \in X, \boldsymbol{E} \neq 0$ and a real number $\lambda$ such that

$$
(\operatorname{curl} \boldsymbol{E}, \operatorname{curl} \boldsymbol{v})=\lambda(\boldsymbol{E}, \boldsymbol{v}), \forall \boldsymbol{v} \in X .
$$


Let us reformulate this problem in a more operator form. More precisely, introduce the operator

$$
A: L^{2}(\Omega)^{3} \rightarrow X: \boldsymbol{u} \rightarrow A \boldsymbol{u},
$$

where $A \boldsymbol{u}$ is the unique element in $X$ that satisfies

$$
(\operatorname{curl} A \boldsymbol{u}, \operatorname{curl} \boldsymbol{v})=(\boldsymbol{u}, \boldsymbol{v}), \forall \boldsymbol{v} \in X .
$$

This problem is meaningful since the bilinear form $a(\boldsymbol{u}, \boldsymbol{v})=(\operatorname{curl} \boldsymbol{u}, \operatorname{curl} \boldsymbol{v})$ is coercive on $X[22,25]$ and therefore problem (5) has a unique solution by the Lax-Milgram Lemma.

Using the operator $A$, the eigenvalue problem (4) is equivalent to the problem of finding $\boldsymbol{E} \in X, \boldsymbol{E} \neq 0$ such that

$$
A \boldsymbol{E}=\mu \boldsymbol{E},
$$

with $\mu=\frac{1}{\lambda}$.

Let us finish this introduction with some notation used in the whole paper: For shortness the $L^{2}(D)^{3}$-norm will be denoted by $\|\cdot\|_{D}$. In the case $D=\Omega$, we will drop the index $\Omega$. The usual norm and seminorm of $H^{t}(D)$ are denoted by $\|\cdot\|_{t, D}$ and $|\cdot|_{t, D}$.

\section{Discontinuous Galerkin discretization}

In this section, we introduce the interior penalty DG discretization of problem (4). To this end, let us introduce the next notation.

The domain $\Omega$ is discretized by a discrete family of conforming meshes $\mathcal{T}_{h}, h \in \Lambda$, made of tetrahedra. The discrete set $\Lambda$ is equal to $\left\{h_{n}: n \in \mathbb{N}\right\}$, where we suppose that the sequence $\left(h_{n}\right)_{n \in \mathbb{N}}$ is strictly decreasing and satisfies $h_{n}>0$, for all $n$ and $h_{n} \rightarrow 0$ as $n \rightarrow \infty$. In other words the meshes are built progressively and become finer and finer.

The family is supposed to be regular in Ciarlet's sense [12], i.e. there exists $\sigma>0$ such that the ratio

$$
\frac{h_{T}}{\rho_{T}} \leq \sigma, \forall T \in \mathcal{T}_{h}, h \in \Lambda,
$$

where we recall that $h_{T}$ is the diameter of $T$ and $\rho_{T}$ is the diameter of the largest ball inscribed into $T$. As usual $h=\max _{T \in \mathcal{T}_{h}} h_{T}$.

As in [26], we further assume that the meshes are weakly quasi-uniform, i.e., if

$$
h_{\text {min }}=\min _{T \in \mathcal{T}_{h}} h_{T},
$$

then there exists $\mu^{\star} \in(0,1)$ such that

$$
h h_{\text {min }}^{-\mu^{\star}} \rightarrow 0 \text { as } h \rightarrow 0 .
$$

As pointed out in [26], quasi-uniform meshes are weakly quasi-uniform. Moreover graded meshes of Raugel's type used in the presence of corner and edge singularities (see for instance [2]) are also weakly quasi-uniform.

Elements will be denoted by $T$, its faces are denoted by $E$. The set of all (interior and boundary) faces of the mesh will be denoted by $\mathcal{E}$. The measure of an element or face is denoted by $|T|:=\operatorname{meas}_{3}(T)$ and $|E|:=\operatorname{meas}_{2}(E)$, respectively. For any face $E$ we will denote by $h_{E}$ its diameter (which is equivalent to the diameter $h_{T}$ of any element $T$ containing $E$ by the regularity of the mesh). For each element $T \in \mathcal{T}_{h}$, denote by $\boldsymbol{n}_{T}$ the unit outward normal vector along $\partial T$.

For our further analysis we need to define some jumps and means through any $E \in \mathcal{E}$ of the mesh. For $E \in \mathcal{E}$ such that $E \subset \Omega$, denote by $T^{+}$and $T^{-}$the two elements of $\mathcal{T}_{h}$ containing $E$. Let $\boldsymbol{v}$ be a vector-valued function 
defined on $T^{+} \cup T^{-}$, and which is in $H^{1}$ inside each element $T^{ \pm}$. We denote by $\boldsymbol{v}^{ \pm}$the traces of $\boldsymbol{v}$ on $E$ taken from $T^{ \pm}$, respectively. Then we define the mean of $\boldsymbol{v}$ on $E$ by

$$
\{\{\boldsymbol{v}\}\}=\frac{\boldsymbol{v}^{+}+\boldsymbol{v}^{-}}{2},
$$

while its tangential jump on $E$ is defined as follows:

$$
\underline{[[v]]_{T}}=\boldsymbol{n}_{T^{+}} \times \boldsymbol{v}^{+}+\boldsymbol{n}_{T^{-}} \times \boldsymbol{v}^{-} .
$$

For a boundary face $E$, i.e., $E \subset \partial \Omega$, there exists a unique element $T^{+} \in \mathcal{T}_{h}$ such that $E \subset \partial T^{+}$. Therefore the mean and the tangential jumps are defined as before by taking $\boldsymbol{v}^{-}=0$.

Following [19], we consider the following discontinuous Galerkin approximation of the continuous eigenvalue problem: Given a mesh $\mathcal{T}_{h}$ and a polynomial degree $k \geq 1$, we consider the approximation space

$$
V_{h}=\left\{v \in L^{2}(\Omega)^{3}: v_{\mid T} \in \mathbb{P}^{k}(T)^{3}, \forall T \in \mathcal{T}_{h}\right\},
$$

where $\mathbb{P}^{k}(T)$ denotes the space of polynomials of total degree at most $k$ on $T$.

On this space, we define the discontinuous curl operator $\operatorname{curl}_{h}$ :

$$
\left(\operatorname{curl}_{h} \boldsymbol{u}\right)_{\mid T}=\operatorname{curl}\left(\boldsymbol{u}_{\mid T}\right), \forall T \in \mathcal{T}_{h},
$$

and the bilinear form $a_{h}(.,$.$) :$

$$
\begin{aligned}
a_{h}(\boldsymbol{u}, \boldsymbol{v}):= & \left(\operatorname{curl}_{h} \boldsymbol{u}, \operatorname{curl}_{h} \boldsymbol{v}\right)-\int_{\mathcal{E}} \underline{\llbracket \boldsymbol{u} \rrbracket_{T}} \cdot\left\{\left\{\operatorname{curl}_{h} \boldsymbol{v}\right\}\right\} \\
& -\int_{\mathcal{E}} \underline{\llbracket \boldsymbol{v} \rrbracket_{T}} \cdot\left\{\left\{\operatorname{curl}_{h} \boldsymbol{u}\right\}\right\}+\alpha \int_{\mathcal{E}} \mathrm{h}^{-1} \underline{\llbracket \boldsymbol{u} \rrbracket_{T}} \cdot \underline{[\boldsymbol{v}]_{T}},
\end{aligned}
$$

where the positive parameter $\alpha$ is the interior penalty stabilization parameter and that will be chosen large enough to ensure coerciveness of the bilinear form $a_{h}$ (see Lem. 5.1 below). Here we use the notation

$$
\int_{\mathcal{E}} \varphi:=\sum_{E \in \mathcal{E}} \int_{E} \varphi(x) \mathrm{d} s(x),
$$

and $\mathrm{h}$ is the function defined on each face $E \in \mathcal{E}$ by

$$
\mathrm{h}(x)=h_{E}, \forall x \in E .
$$

The discontinuous Galerkin approximation of the eigenvalue problem (4) reads now: Find $\boldsymbol{E}_{h} \in V_{h}, \boldsymbol{E}_{h} \neq 0$ and $\lambda_{h} \in \mathbb{R}$ such that

$$
a_{h}\left(\boldsymbol{E}_{h}, \boldsymbol{v}_{h}\right)=\lambda_{h}\left(\boldsymbol{E}_{h}, \boldsymbol{v}_{h}\right), \forall \boldsymbol{v}_{h} \in V_{h} .
$$

\section{The Discrete Friedrichs INEQUALITY}

Following $[19,20]$ we consider the space

$$
V(h)=\mathcal{H}_{0}(\operatorname{curl} ; \Omega)+V_{h},
$$

that we equipped with the norm

$$
\|\boldsymbol{u}\|_{D G, h}^{2}=\|\boldsymbol{u}\|^{2}+|\boldsymbol{u}|_{D G, h}^{2},
$$


where the semi-norm is defined by

$$
|\boldsymbol{u}|_{D G, h}^{2}=\left\|\operatorname{curl}_{h} \boldsymbol{u}\right\|^{2}+\int_{\mathcal{E}} \mathrm{h}^{-1}\left|\underline{[\underline{u}]]_{T}}\right|^{2} .
$$

Now we consider $V_{h}^{c}=V_{h} \cap \mathcal{H}_{0}(\operatorname{curl} ; \Omega)$ and decompose $V_{h}$ into

$$
V_{h}=V_{h}^{c} \oplus V_{h}^{\perp}
$$

where the decomposition is orthogonal with respect to the following inner product

$$
(\boldsymbol{u}, \boldsymbol{v})_{D G, h \text { new }}=h^{-2}(\boldsymbol{u}, \boldsymbol{v})+\left(\operatorname{curl}_{h} \boldsymbol{u}, \operatorname{curl}_{h} \boldsymbol{v}\right)+\int_{\mathcal{E}} \mathrm{h}^{-1} \underline{[\boldsymbol{u}]]_{T}} \cdot \underline{[\boldsymbol{v}]]_{T}} \cdot
$$

In other words, $V_{h}^{\perp}$ is the orthogonal complement of $V_{h}^{c}$ into $V_{h}$ for this inner product. Note that our orthogonal decomposition is different from the one from $[19,20]$ but is motivated by the following result proved in Proposition 4.5 of [19] (or in the Appendix of [20]):

Theorem 4.1. There exists a positive constant $\mathrm{C}$ (independent of $h$ ) such that for any $\boldsymbol{v} \in V_{h}$, there exists $v^{c} \in V_{h}^{c}$ satisfying

$$
\begin{gathered}
\left\|\boldsymbol{v}-\boldsymbol{v}^{c}\right\|_{D G, h} \leq \mathrm{C}\left(\int_{\mathcal{E}} \mathrm{h}^{-1}\left|\underline{[[\boldsymbol{v}]]_{T}}\right|^{2}\right)^{1 / 2}, \\
\left\|\boldsymbol{v}-\boldsymbol{v}^{c}\right\| \leq \mathrm{C} h\left(\int_{\mathcal{E}} \mathrm{h}^{-1}\left|\underline{[\underline{[v}]]_{T}}\right|^{2}\right)^{1 / 2} .
\end{gathered}
$$

The following approximation property and norm equivalence (compare with Prop. 4.6 from [19] and Thm. 2 of [20]) will be the basis of our analysis below.

Corollary 4.2. There exists a positive constant $\mathrm{C}$ (independent of $h$ ) such that for all $\boldsymbol{v} \in V_{h}$, the orthogonal projection $\pi_{h} \boldsymbol{v}$ of $\boldsymbol{v}$ on $V_{h}^{c}$ wrt to the inner product $(\cdot, \cdot)_{D G, h \text { new satisfies }}$

$$
\left.\left\|\boldsymbol{v}-\pi_{h} \boldsymbol{v}\right\|_{D G, h \text { new }} \leq \mathrm{C}\left(\int_{\mathcal{E}} \mathrm{h}^{-1}\left|\underline{[[\boldsymbol{v}]]_{T}}\right|^{2}\right)\right)^{1 / 2}
$$

where $\|\cdot\|_{D G, h \text { new }}=(\cdot, \cdot)_{D G, h \text { new }}^{1 / 2}$.

Proof. The requested estimate directly follows from Theorem 4.1 because it shows that there exists $\boldsymbol{v}^{c} \in V_{h}^{c}$ such that

$$
\left.\left\|\boldsymbol{v}-\boldsymbol{v}^{c}\right\|_{D G, h \text { new }} \leq \mathrm{C}\left(\int_{\mathcal{E}} \mathrm{h}^{-1}\left|\underline{[\underline{v}]]_{T}}\right|^{2}\right)\right)^{1 / 2},
$$

and clearly $\left\|\boldsymbol{v}-\pi_{h} \boldsymbol{v}\right\|_{D G, h \text { new }} \leq\left\|\boldsymbol{v}-\boldsymbol{v}^{c}\right\|_{D G, h \text { new }}$

Note that this corollary shows in particular that

$$
\left.\left\|\boldsymbol{v}-\pi_{h} \boldsymbol{v}\right\| \leq \mathrm{C} h\left(\int_{\mathcal{E}} \mathrm{h}^{-1}\left|\underline{\left[[\boldsymbol{v}]_{T}\right.}\right|^{2}\right)\right)^{1 / 2}
$$

which is not the case if we take the orthogonal projection wrt the natural inner product associated with $\|\cdot\|_{D G, h}$. 
Corollary 4.3. There exists a positive constant $\mathrm{C}$ (independent of $h$ ) such that

$$
\mathrm{C}\left\|\boldsymbol{v}_{h}\right\|_{D G, h} \leq\left\|\boldsymbol{v}_{h}\right\|_{V_{h}^{\perp}} \leq\left\|\boldsymbol{v}_{h}\right\|_{D G, h}, \forall \boldsymbol{v}_{h} \in V_{h}^{\perp},
$$

where the norm $\|\cdot\|_{V_{h}^{\perp}}$ is defined

$$
\left\|\boldsymbol{v}_{h}\right\|_{V_{h}^{\perp}}^{2}:=\int_{\mathcal{E}} \mathrm{h}^{-1}\left|\underline{\left[\left[\boldsymbol{v}_{h}\right]\right]_{T}}\right|^{2}, \forall \boldsymbol{v}_{h} \in V_{h}^{\perp} .
$$

Proof. For $\boldsymbol{v}_{h} \in V_{h}^{\perp}$, its projection $\pi_{h} \boldsymbol{v}_{h}=0$ and by Corollary 4.2 , we obtain

$$
\left\|\boldsymbol{v}_{h}\right\|_{D G, h \text { new }} \leq \mathrm{C}\left\|\boldsymbol{v}_{h}\right\|_{V_{h}^{\perp}}
$$

for some $\mathrm{C}>0$. The proof is completed by the simple estimate $\left\|\boldsymbol{v}_{h}\right\|_{D G, h} \leq \max \left\{1, h_{0}\right\}\left\|\boldsymbol{v}_{h}\right\|_{D G, h \text { new }}$.

Let us finally introduce the spaces:

$$
\begin{aligned}
& S_{h}=\left\{p_{h} \in H_{0}^{1}(\Omega): p_{h \mid T} \in \mathbb{P}^{k+1}(T), \forall T \in \mathcal{T}_{h}\right\} \\
& X_{h}=\left\{\boldsymbol{v}_{h} \in V_{h}:\left(\boldsymbol{v}_{h}, \nabla p_{h}\right)=0, \forall p_{h} \in S_{h}\right\}
\end{aligned}
$$

The space $X_{h}$ corresponds to the subspace of $V_{h}$ of functions which are "discrete divergence free". Note further that

$$
\nabla S_{h} \subset V_{h}^{c}
$$

Now we are ready to prove a property which is similar to the standard discrete compactness property.

Theorem 4.4. Let $\left(h_{n}\right)_{n \in \mathbb{N}} \subset \Lambda^{\mathbb{N}}$ satisfy $h_{n} \rightarrow 0$ as $n$ goes to infinity. Let $\left(\boldsymbol{u}_{n}\right)_{n \in \mathbb{N}}$ be a sequence such that $\boldsymbol{u}_{n}$ belongs to $X_{h_{n}}$ and satisfying

$$
\left\|\boldsymbol{u}_{n}\right\|_{D G, h_{n}} \leq \mathrm{C}, \forall n \in \mathbb{N}
$$

for some $\mathrm{C}>0$. Then using the orthogonal decomposition

$$
\boldsymbol{u}_{n}=\boldsymbol{u}_{n}^{c}+\boldsymbol{u}_{n}^{\perp}
$$

from (8), with $\boldsymbol{u}_{n}^{c} \in V_{h_{n}}^{c}$ and $\boldsymbol{u}_{n}^{\perp} \in V_{h_{n}}^{\perp}$. Then

$$
\boldsymbol{u}_{n}^{c} \in X_{h_{n}}^{c}:=\left\{\boldsymbol{v}_{h} \in V_{h_{n}}^{c}:\left(\boldsymbol{v}_{h}, \nabla p_{h_{n}}\right)=0, \forall p_{h_{n}} \in S_{h_{n}}\right\}
$$

and there exists a subsequence, still denoted by $\left(\boldsymbol{u}_{n}\right)_{n \in \mathbb{N}}$, and $\boldsymbol{u} \in X$ such that

$$
\begin{array}{r}
\boldsymbol{u}_{n} \rightarrow \boldsymbol{u} \text { strongly in } L^{2}(\Omega)^{3} \text { as } n \rightarrow \infty, \\
\boldsymbol{u}_{n}^{c} \rightarrow \boldsymbol{u} \text { weakly in } \mathcal{H}_{0}(\operatorname{curl} ; \Omega) \text { as } n \rightarrow \infty .
\end{array}
$$

Proof. As $\boldsymbol{u}_{n} \in X_{h_{n}}$, it satisfies

$$
\left(\boldsymbol{u}_{n}, \nabla q\right)=0, \forall q \in S_{h_{n}}
$$

and by (11), we get

$$
\left(\boldsymbol{u}_{n}^{c}, \nabla q\right)+\left(\boldsymbol{u}_{n}^{\perp}, \nabla q\right)=0, \forall q \in S_{h_{n}} .
$$

For $q \in S_{h_{n}}$, by (9) we have $\operatorname{curl}_{h_{n}} \nabla q=\operatorname{curl} \nabla q=0$ and $\underline{[\nabla \nabla q]_{T}}=0$ on each edge $E$, therefore

$$
h_{n}^{-2}\left(\boldsymbol{u}_{n}^{\perp}, \nabla q\right)=\left(\boldsymbol{u}_{n}^{\perp}, \nabla q\right)_{D G, h_{n} n e w}=0, \forall q \in S_{h_{n}},
$$


again thanks to (9). The two above identities imply that

$$
\left(\boldsymbol{u}_{n}^{c}, \nabla q\right)=0, \forall q \in S_{h_{n}}
$$

which shows the inclusion (12).

Now the decomposition (11) yields

$$
\left\|\boldsymbol{u}_{n}^{c}\right\|_{D G, h_{n}} \leq\left\|\boldsymbol{u}_{n}\right\|_{D G, h_{n}}+\left\|\boldsymbol{u}_{n}^{\perp}\right\|_{D G, h_{n}}, \forall n \in \mathbb{N},
$$

and by Corollary 4.3, there exists a positive constant C (independent of $h_{n}$ ) such that

$$
\left\|\boldsymbol{u}_{n}^{c}\right\|_{D G, h_{n}} \leq\left\|\boldsymbol{u}_{n}\right\|_{D G, h_{n}}+\mathrm{C}\left\|\boldsymbol{u}_{n}^{\perp}\right\|_{V_{h_{n}}^{\perp}}, \forall n \in \mathbb{N}
$$

As $\underline{\left[\boldsymbol{u}_{n}^{\perp} \rrbracket_{T}\right.}=\underline{\left[\boldsymbol{u}_{n} \rrbracket_{T}\right.}$, we deduce that

$$
\left\|\boldsymbol{u}_{n}^{c}\right\|_{D G, h_{n}} \leq(1+\mathrm{C})\left\|\boldsymbol{u}_{n}\right\|_{D G, h_{n}}, \forall n \in \mathbb{N}
$$

By (10) we then have

$$
\left\|\boldsymbol{u}_{n}^{c}\right\|_{\mathcal{H}_{0}(\operatorname{curl} ; \Omega)}=\left\|\boldsymbol{u}_{n}^{c}\right\|_{D G, h_{n}} \leq \mathrm{C}, \forall n \in \mathbb{N} .
$$

Consequently the sequence $\left(\boldsymbol{u}_{n}^{c}\right)_{n \in \mathbb{N}}$ is bounded in $\mathcal{H}_{0}(\operatorname{curl} ; \Omega)$. Hence Theorem 4.1 of [26] (also valid for the second family of edge elements on tetrahedra, see the beginning of Sect. 3 of [26]) yields a subsequence, still denoted by $\left(\boldsymbol{u}_{n}^{c}\right)_{n \in \mathbb{N}}$, and $\boldsymbol{u} \in X$ satisfying (14) and

$$
\boldsymbol{u}_{n}^{c} \rightarrow \boldsymbol{u} \text { strongly in } L^{2}(\Omega)^{3} \text { as } n \rightarrow \infty .
$$

But according to Corollary 4.2 ,

$$
\left\|\boldsymbol{u}_{n}^{\perp}\right\| \leq C h_{n}\left\|\boldsymbol{u}_{n}\right\|_{D G, h_{n}} \leq C h_{n}
$$

which shows that

$$
\boldsymbol{u}_{n}^{\perp} \rightarrow 0 \text { strongly in } L^{2}(\Omega)^{3} \text { as } n \rightarrow \infty
$$

The property (13) is proved.

This "discontinuous discrete compactness" result allows us to prove the discrete Friedrichs inequality:

Theorem 4.5. There exists a positive constant $\mathrm{C}>0$ such that for all $h \in \Lambda$, one has

$$
\left\|\boldsymbol{u}_{h}\right\| \leq \mathrm{C}\left|\boldsymbol{u}_{h}\right|_{D G, h}, \forall \boldsymbol{u}_{h} \in X_{h} .
$$

Proof. Assume that (17) does not hold, then there exists a sequence $\boldsymbol{u}_{n} \in X_{h_{n}}, n \in \mathbb{N}$ such that

$$
\begin{aligned}
\left\|\boldsymbol{u}_{n}\right\| & =1, \forall n \in \mathbb{N}, \\
\left|\boldsymbol{u}_{n}\right|_{D G, h_{n}} & =\frac{1}{n}, \forall n \in \mathbb{N} .
\end{aligned}
$$

By the definition of the semi-norm $|\cdot|_{D G, h_{n}}$, we then have

$$
\int_{\mathcal{E}} \mathrm{h}^{-1}\left|\underline{\llbracket \boldsymbol{u}_{n} \rrbracket_{T}}\right|^{2} \leq \frac{1}{n^{2}}
$$


Using the (orthogonal) decomposition (11) of $\boldsymbol{u}_{n}$, we obtain

$$
\int_{\mathcal{E}} \mathrm{h}^{-1}\left|\underline{\left[\left[\boldsymbol{u}_{n}^{\perp}\right]\right]_{T}}\right|^{2} \leq \frac{1}{n^{2}}
$$

because $\underline{\left.\llbracket \boldsymbol{u}_{n}^{c}\right]_{T}}=0$. Now with the help of Corollary 4.3, we deduce that

$$
\left\|\boldsymbol{u}_{n}^{\perp}\right\|_{D G, h_{n}} \leq \frac{\mathrm{C}_{1}}{n}
$$

for some positive constant $\mathrm{C}_{1}$.

From (18) and (19), the sequence $\left(\boldsymbol{u}_{n}\right)_{n \in \mathbb{N}}$ satisfies

$$
\left\|\boldsymbol{u}_{n}\right\|_{D G, h_{n}} \leq \mathrm{C}_{2}, \forall n \in \mathbb{N}
$$

for some positive constant $\mathrm{C}_{2}$. Therefore by Theorem 4.4, there exists a subsequence, still denoted by $\left(\boldsymbol{u}_{n}\right)_{n \in \mathbb{N}}$, and $\boldsymbol{u} \in X$ satisfying (13) and (14).

By (13) and (18), we deduce that

$$
\|\boldsymbol{u}\|=1
$$

Now from (14), we may write

$$
\|\operatorname{curl} u\| \leq \lim _{n \rightarrow \infty}\left\|\operatorname{curl} \boldsymbol{u}_{n}^{c}\right\|
$$

But the triangular inequality leads to

$$
\begin{aligned}
\left\|\operatorname{curl} \boldsymbol{u}_{n}^{c}\right\| & \leq\left\|\operatorname{curl}_{h_{n}} \boldsymbol{u}_{n}\right\|+\left\|\operatorname{curl}_{h_{n}} \boldsymbol{u}_{n}^{\perp}\right\| \\
& \leq\left|\boldsymbol{u}_{n}\right|_{D G, h_{n}}+\left\|\boldsymbol{u}_{n}^{\perp}\right\|_{D G, h_{n}}
\end{aligned}
$$

and by (19) and (20), we conclude that

$$
\left\|\operatorname{curl} \boldsymbol{u}_{n}^{c}\right\| \rightarrow 0 \text { as } n \rightarrow \infty
$$

Hence

$$
\operatorname{curl} \boldsymbol{u}=0 \text { in } \Omega \text {, }
$$

and since $\boldsymbol{u}$ belongs to $X, \boldsymbol{u}=0$ by the standard Friedrichs inequality. This is a contradiction with (21).

\section{The Discrete eigenvalue PRoblem}

Now we can come back to the discrete eigenvalue problem (7) and show its well posedness.

Lemma 5.1. There exists a parameter $\alpha_{\min }>0$, independent of the mesh size $h$ such that for $\alpha \geq \alpha_{\min }>0$, and $h \in \Lambda$, the bilinear form $a_{h}$ is strongly coercive on $X_{h}$, namely there exists $\beta>0$ independent of the mesh size $h$ such that

$$
a_{h}\left(\boldsymbol{u}_{h}, \boldsymbol{u}_{h}\right) \geq \beta\left\|\boldsymbol{u}_{h}\right\|_{D G, h}^{2}, \forall \boldsymbol{u}_{h} \in X_{h} .
$$

Proof. Using standard inverse estimates (see Lem. 3.1 of [19]), for $\alpha \geq \alpha_{\min }>0$, there exists $\beta_{0}>0$ independent of the mesh size $h$ such that

$$
a_{h}\left(\boldsymbol{u}_{h}, \boldsymbol{u}_{h}\right) \geq \beta_{0}\left|\boldsymbol{u}_{h}\right|_{D G, h}^{2}, \forall \boldsymbol{u}_{h} \in V_{h} .
$$

The conclusion follows from Theorem 4.5.

In view of this lemma, we reduce problem (7) to the space $X_{h}$. 
Lemma 5.2. If $\lambda_{h} \neq 0$, then (7) is equivalent to find $\boldsymbol{E}_{h} \in X_{h}$ solution of

$$
a_{h}\left(\boldsymbol{E}_{h}, \boldsymbol{v}_{h}\right)=\lambda_{h}\left(\boldsymbol{E}_{h}, \boldsymbol{v}_{h}\right), \forall \boldsymbol{v}_{h} \in X_{h} .
$$

Proof. $\Rightarrow$ Let $\boldsymbol{E}_{h} \in V_{h}$ be a solution of (7). We only need to show that it belongs to $X_{h}$. To this end, we simply take $\boldsymbol{v}_{h}=\nabla q_{h}$ with $q_{h} \in S_{h}$ as test function in (7) to obtain

$$
a_{h}\left(\boldsymbol{E}_{h}, \nabla q_{h}\right)=\lambda_{h}\left(\boldsymbol{E}_{h}, \nabla q_{h}\right) .
$$

Since $a_{h}\left(\boldsymbol{E}_{h}, \nabla q_{h}\right)=0$ and $\lambda_{h} \neq 0$, we conclude that

$$
\left(\boldsymbol{E}_{h}, \nabla q_{h}\right)=0, \forall q_{h} \in S_{h} .
$$

$\Leftarrow$ Let $\boldsymbol{E}_{h} \in X_{h}$ be a solution of (22). As $X_{h}$ is a closed subspace of $V_{h}$, any $\boldsymbol{v}_{h} \in V_{h}$ may be split up

$$
\boldsymbol{v}_{h}=\boldsymbol{v}_{h}^{0}+\nabla q_{h},
$$

where $\boldsymbol{v}_{h}^{0}$ belongs to $X_{h}$ and $q_{h}$ in $S_{h}$. Consequently, we obtain

$$
\begin{aligned}
a_{h}\left(\boldsymbol{E}_{h}, \boldsymbol{v}_{h}\right) & =a_{h}\left(\boldsymbol{E}_{h}, \boldsymbol{v}_{h}^{0}\right)=\lambda_{h}\left(\boldsymbol{E}_{h}, \boldsymbol{v}_{h}^{0}\right) \\
& =\lambda_{h}\left\{\left(\boldsymbol{E}_{h}, \boldsymbol{v}_{h}\right)-\left(\boldsymbol{E}_{h}, \nabla q_{h}\right)\right\}=\lambda_{h}\left(\boldsymbol{E}_{h}, \boldsymbol{v}_{h}\right),
\end{aligned}
$$

using successively the property $a_{h}\left(\boldsymbol{E}_{h}, \nabla q_{h}\right)=0$, the identity (22) and the fact that $\boldsymbol{E}_{h} \in X_{h}$.

From Lemma 5.1, the eigenvalues problem (22) has only positive eigenvalues. Hence from Lemma 5.2, problem (7) has only nonegative eigenvalues. Thus from the computational point of view, we can solve problem (7) and keep the positive eigenvalues, since only these ones have a physical meaning. Owing to Lemma 5.2, our theoretical analysis can be based on (22).

As in the continuous case, we rewrite problem (22) as a discrete operator equation. Namely we introduce the discrete operator

$$
A_{h}: L^{2}(\Omega)^{3} \rightarrow X_{h}: \boldsymbol{u} \rightarrow A_{h} \boldsymbol{u},
$$

where $A_{h} \boldsymbol{u} \in X_{h}$ is the unique solution of

$$
a_{h}\left(A_{h} \boldsymbol{u}, \boldsymbol{v}_{h}\right)=\left(\boldsymbol{u}, \boldsymbol{v}_{h}\right), \forall \boldsymbol{v}_{h} \in X_{h} .
$$

Thanks to Lemma 5.1 this problem is well-posed and hence $A_{h}$ is well-defined.

Lemma 5.3. $\boldsymbol{E}_{h} \in X_{h}$ is solution of (22) for $\lambda_{h}>0$ if and only if $E_{h} \in L^{2}(\Omega)^{3}$ satisfies

$$
A_{h} \boldsymbol{E}_{h}=\mu_{h} \boldsymbol{E}_{h},
$$

with $\mu_{h}=\frac{1}{\lambda_{h}}$.

Proof. $\Rightarrow$ Let $\boldsymbol{E}_{h} \in X_{h}$ be a solution of (22). By the definition of $A_{h}$, we may write for any $\boldsymbol{v}_{h} \in X_{h}$

$$
a_{h}\left(A_{h} \boldsymbol{E}_{h}, \boldsymbol{v}_{h}\right)=\left(\boldsymbol{E}_{h}, \boldsymbol{v}_{h}\right)=\frac{1}{\lambda_{h}} a_{h}\left(\boldsymbol{E}_{h}, \boldsymbol{v}_{h}\right) .
$$

This leads to the conclusion since $a_{h}(\cdot, \cdot)$ is an inner product on $X_{h}$.

$\Leftrightarrow$ Let $\boldsymbol{E}_{h} \in L^{2}(\Omega)^{3}$ be a solution of $(24)$. Then $\boldsymbol{E}_{h}=\frac{1}{\mu_{h}} A_{h} \boldsymbol{E}_{h}$, which belongs to $X_{h}$. The conclusion then follows from this identity and (23). 


\section{Collective compactness and pointwise COnvergence}

Following [26], we prove the convergence of the positive eigenvalues of the discrete problem (7) to the positive eigenvalues of the continuous problem (4) by using the theory of collectively compact and pointwise convergent operators $[1,11,27]$. For completeness, we first recall a basis result that will be used later on.

Definition 6.1. Let $X, Y$ be two normed spaces and $\Lambda$ a subset of $\mathbb{R}$. A set $\mathcal{A}=\left\{A_{h}: X \rightarrow Y, h \in \Lambda\right\}$ of linear operators from $X$ to $Y$ is called collectively compact if for each bounded set $U$ of $X$, the range

$$
\mathcal{A}(U):=\left\{A_{h} u: u \in U, h \in \Lambda\right\}
$$

is relatively compact in $Y$.

With this definition, we can recall the next convergence result proved in [27].

Theorem 6.2. Let $X$ be a Hilbert space of inner product $(\cdot, \cdot)_{X}$ and norm $\|\cdot\|_{X}$, and let $\Lambda=\left\{h_{n}: n \in \mathbb{N}\right\}$ be a discrete subset of $\mathbb{R}$ such that $h_{n} \rightarrow 0$ as $n \rightarrow \infty$. Assume that the set $\mathcal{A}=\left\{A_{h}: X \rightarrow X, h \in \Lambda\right\}$ of linear selfadjoint operators in $X$ is collectively compact. Assume furthermore that there exists a selfadjoint and compact operator $A$ in $X$ such that $A_{h}$ converges pointwise to $A$ (i.e., for all $x \in X, A_{h} x \rightarrow A x$ in $X$ as $h \rightarrow 0$ ). Let $\mu$ be an eigenvalue of $A$ of multiplicity $m$ and denote by $\left\{\phi_{j}\right\}_{j=1}^{m}$ the set of associated orthogonal eigenvectors. Then for any $\varepsilon>0$ such that the disk $B(\mu, \varepsilon)$ of radius $\varepsilon$ and center $\mu$ contains no other eigenvalues of $A$, there exists $h_{\varepsilon}$ such that for all $h<h_{\varepsilon}, A_{h}$ has exactly $m$ eigenvalues (repeated according to their multiplicity) in $B(\mu, \varepsilon)$. Moreover for $h<h_{\varepsilon}$, if we denote by $\mu_{h, j}, j=1, \ldots, m$, the set of the eigenvalues of $A_{h}$ in $B(\mu, \varepsilon)$, then for all $j=1, \ldots, m$, there exists a positive constant $\mathrm{C}$ such that

$$
\left.\left|\mu-\mu_{h, j}\right| \leq \mathrm{C}\left(\sum_{j, l=1}^{m} \mid\left(\left(A-A_{h}\right) \phi_{j}, \phi_{l}\right)\right)_{X} \mid+\left(\sum_{j=1}^{m}\left\|\left(A-A_{h}\right) \phi_{j}\right\|_{X}^{2}\right)^{1 / 2}\right) .
$$

The remainder of the section consists in checking that the set of operators $A_{h}$ defined by (23) satisfies the hypotheses of the above theorem.

Theorem 6.3. The set $\mathcal{A}:=\left\{A_{h}: h \in \Lambda\right\}$ is collectively compact.

Proof. Let $U$ be a bounded set of $L^{2}(\Omega)^{3}$. By the definition of $A_{h}$, for any $\boldsymbol{u} \in U, A_{h} \boldsymbol{u} \in X_{h}$ satisfies

$$
a_{h}\left(A_{h} \boldsymbol{u}, \boldsymbol{v}_{h}\right)=\left(\boldsymbol{u}, \boldsymbol{v}_{h}\right), \forall \boldsymbol{v}_{h} \in X_{h}
$$

By Lemma 5.1, we then have

$$
\left\|A_{h} \boldsymbol{u}\right\|_{D G, h}^{2} \leq \beta^{-1} a_{h}\left(A_{h} \boldsymbol{u}, A_{h} \boldsymbol{u}\right)=\beta^{-1}\left(\boldsymbol{u}, A_{h} \boldsymbol{u}\right) .
$$

By Cauchy-Schwarz's inequality and the boundedness assumption of $U$, we obtain

$$
\left\|A_{h} \boldsymbol{u}\right\|_{D G, h} \leq \mathrm{C}, \forall h \in \Lambda,
$$

for some $C>0$ (independent of $h$ ).

This estimate and Theorem 4.4 imply that the

$$
W=\left\{A_{h} \boldsymbol{u}: \boldsymbol{u} \in U, h \in \Lambda\right\},
$$

is precompact in $L^{2}(\Omega)^{3}$. Indeed let us fix a sequence $\left(A_{h_{n}} \boldsymbol{u}_{n}\right)_{n \in \mathbb{N}}$ in $W$. Then either $h_{n} \rightarrow 0$ as $n \rightarrow \infty$ and since (26) guarantees that (10) holds, we conclude by Theorem 4.4; or $h_{n}$ does not tend to zero as $n \rightarrow \infty$ and in that case the set $\left\{h_{n}: n \in \mathbb{N}\right\}$ is finite, in this last case we conclude by finite dimensionality. 
Let us go on with the pointwise convergence:

Theorem 6.4. Let $\boldsymbol{u} \in L^{2}(\Omega)^{3}$, then

$$
A_{h} \boldsymbol{u} \rightarrow \text { Au strongly in } L^{2}(\Omega)^{3} \text {, as } h \rightarrow 0,
$$

where we recall that $A$ was defined by (5).

Proof. We follow the proof of Theorem 4.5 of [26] with the necessary adaptations due to the discontinuous scheme. First following some recent ideas from $[4,19,20]$, we introduce the lifting operator

$$
\mathcal{L}: V(h) \rightarrow V_{h}: \boldsymbol{v} \rightarrow \mathcal{L}(\boldsymbol{v})
$$

where $\mathcal{L}(\boldsymbol{v})$ is the unique element in $V_{h}$ such that

$$
\left(\mathcal{L}(\boldsymbol{v}), \boldsymbol{w}_{h}\right)=\int_{\mathcal{E}} \underline{\left[\left[\boldsymbol{v} \rrbracket_{T}\right.\right.} \cdot\left\{\left\{\boldsymbol{w}_{h}\right\}\right\}, \forall \boldsymbol{w}_{h} \in V_{h} .
$$

Introduce further the modified bilinear form

$$
\begin{aligned}
\tilde{a}_{h}(\boldsymbol{u}, \boldsymbol{v}) & :=\left(\operatorname{curl}_{h} \boldsymbol{u}, \operatorname{curl}_{h} \boldsymbol{v}\right)-\left(\mathcal{L}(\boldsymbol{u}), \operatorname{curl}_{h} \boldsymbol{v}\right) \\
& -\left(\mathcal{L}(\boldsymbol{v}), \operatorname{curl}_{h} \boldsymbol{u}\right)+\alpha \int_{\mathcal{E}} \mathrm{h}^{-1} \underline{[\underline{u}]_{T}} \cdot \underline{[\underline{\boldsymbol{v}}]_{T}} .
\end{aligned}
$$

We may remark that

$$
\begin{aligned}
\tilde{a}_{h}\left(\boldsymbol{u}_{h}, \boldsymbol{v}_{h}\right) & =a_{h}\left(\boldsymbol{u}_{h}, \boldsymbol{v}_{h}\right), \forall \boldsymbol{u}_{h}, \boldsymbol{v}_{h} \in V_{h}, \\
\tilde{a}_{h}(\boldsymbol{u}, \boldsymbol{v}) & =(\operatorname{curl} \boldsymbol{u}, \operatorname{curl} \boldsymbol{v}), \forall \boldsymbol{u}, \boldsymbol{v} \in \mathcal{H}_{0}(\operatorname{curl} ; \Omega) .
\end{aligned}
$$

Now we transform the discrete problem (23) and the continuous problem (5) in mixed problems: if $A_{h} \boldsymbol{u} \in X_{h}$ is solution of (23), then the pair $\left(A_{h} \boldsymbol{u}, p_{h}\right) \in V_{h} \times S_{h}$ is the unique solution of

$$
\left\{\begin{array}{l}
a_{h}\left(A_{h} \boldsymbol{u}, \boldsymbol{v}_{h}\right)+b\left(\boldsymbol{v}_{h}, p_{h}\right)=\left(\boldsymbol{u}, \boldsymbol{v}_{h}\right), \forall \boldsymbol{v}_{h} \in V_{h}, \\
b\left(A_{h} \boldsymbol{u}, q_{h}\right)=0, \forall q_{h} \in S_{h}
\end{array}\right.
$$

where $b(\boldsymbol{v}, p)=(\nabla p, \boldsymbol{v})$. Indeed the second identity means that $A_{h} \boldsymbol{u} \in X_{h}$ and the first one reduces to (23), when the test functions are taken in $X_{h}$ because $b\left(\boldsymbol{v}_{h}, p_{h}\right)=0$ for $p_{h} \in S_{h}$ and $\boldsymbol{v}_{h} \in X_{h}$. This mixed problem has a unique solution because $a_{h}$ is coercive on $X_{h}$, which is precisely the kernel of $b$ in $V_{h}$, and $b$ satisfies the discrete inf-sup condition

$$
\sup _{\boldsymbol{v}_{h} \in V_{h}} \frac{b\left(\boldsymbol{v}_{h}, p_{h}\right)}{\left\|\boldsymbol{v}_{h}\right\|_{D G, h}} \geq\left|p_{h}\right|_{1, \Omega}
$$

for all $p_{h} \in S_{h}$, since $\boldsymbol{v}_{h}=\nabla p_{h}$ yields

$$
\frac{b\left(\boldsymbol{v}_{h}, p_{h}\right)}{\left\|\boldsymbol{v}_{h}\right\|_{D G, h}}=\left|p_{h}\right|_{1, \Omega} .
$$

Note that in the above problem we can replace $a_{h}$ by $\tilde{a}_{h}$.

In a similar manner if $A \boldsymbol{u} \in X$ is solution of (5), then the pair $(A \boldsymbol{u}, p) \in \mathcal{H}_{0}(\operatorname{curl} ; \Omega) \times H_{0}^{1}(\Omega)$ is the unique solution of

$$
\left\{\begin{array}{l}
(\operatorname{curl} A \boldsymbol{u}, \operatorname{curl} \boldsymbol{v})+b(\boldsymbol{v}, p)=(\boldsymbol{u}, \boldsymbol{v}), \forall \boldsymbol{v} \in \mathcal{H}_{0}(\operatorname{curl} ; \Omega) \\
b(A \boldsymbol{u}, q)=0, \forall q \in H_{0}^{1}(\Omega)
\end{array}\right.
$$


Since $V_{h}$ is not included into $\mathcal{H}_{0}(\operatorname{curl} ; \Omega)$, we may look at $(27)$ as a nonconforming approximation of (28). Therefore by Proposition II.2.16 of [10], we may write

$$
\begin{aligned}
\left\|A \boldsymbol{u}-A \boldsymbol{u}_{h}\right\|_{D G, h} \leq & \mathrm{C}\left(\inf _{\boldsymbol{v}_{h} \in V_{h}}\left\|A \boldsymbol{u}-\boldsymbol{v}_{h}\right\|_{D G, h}+\inf _{p_{h} \in S_{h}}\left|p-p_{h}\right|_{1, \Omega}\right. \\
& \left.+\sup _{\boldsymbol{w}_{h} \in V_{h}} \frac{\left|\tilde{a}_{h}\left(A \boldsymbol{u}, \boldsymbol{w}_{h}\right)+b\left(\boldsymbol{w}_{h}, p\right)-\left(\boldsymbol{u}, \boldsymbol{w}_{h}\right)\right|}{\left\|\boldsymbol{w}_{h}\right\|_{D G, h}}\right) .
\end{aligned}
$$

The two first terms of this right-hand side are so-called approximation error terms and the third one is the consistency error term.

Since $V_{h}^{c}$ is included into $V_{h}$ we can estimate the first approximation error term by

$$
\inf _{\boldsymbol{v}_{h} \in V_{h}}\left\|A \boldsymbol{u}-\boldsymbol{v}_{h}\right\|_{D G, h} \leq \inf _{\boldsymbol{v}_{h} \in V_{h}^{c}}\left\|A \boldsymbol{u}-\boldsymbol{v}_{h}\right\|_{D G, h}=\inf _{\boldsymbol{v}_{h} \in V_{h}^{c}}\left\|A \boldsymbol{u}-\boldsymbol{v}_{h}\right\|_{\mathcal{H}_{0}(\mathrm{curl} ; \Omega)} .
$$

Since $V_{h}^{c}$ is dense in $\mathcal{H}_{0}(\operatorname{curl} ; \Omega)$ (see Thm. 4.5 of [26]) the above right-hand side tends to zero as $h \rightarrow 0$, hence

$$
\inf _{\boldsymbol{v}_{h} \in V_{h}}\left\|A \boldsymbol{u}-\boldsymbol{v}_{h}\right\|_{D G, h} \rightarrow 0 \text { as } h \rightarrow 0 .
$$

Similarly the density of $S_{h}$ in $H_{0}^{1}(\Omega)$ leads to

$$
\inf _{p_{h} \in S_{h}}\left|p-p_{h}\right|_{1, \Omega} \rightarrow 0 \text { as } h \rightarrow 0 .
$$

It remains to estimate the consistency term. Let us denote by

$$
r_{h}\left(\boldsymbol{w}_{h}\right)=\tilde{a}_{h}\left(A \boldsymbol{u}, \boldsymbol{w}_{h}\right)+b\left(\boldsymbol{w}_{h}, p\right)-\left(\boldsymbol{u}, \boldsymbol{w}_{h}\right) .
$$

By the definition of $\tilde{a}_{h}$, it can be written

$$
r_{h}\left(\boldsymbol{w}_{h}\right)=\left(\operatorname{curl} A \boldsymbol{u}, \operatorname{curl}_{h} \boldsymbol{w}_{h}\right)-\left(\mathcal{L}\left(\boldsymbol{w}_{h}\right), \operatorname{curl} A \boldsymbol{u}\right)+b\left(\boldsymbol{w}_{h}, p\right)-\left(\boldsymbol{u}, \boldsymbol{w}_{h}\right) .
$$

Introducing $\Pi_{h}$ the $L^{2}$-orthogonal projection on $V_{h}$, we may write

$$
r_{h}\left(\boldsymbol{w}_{h}\right)=\left(\operatorname{curl} A \boldsymbol{u}, \operatorname{curl}_{h} \boldsymbol{w}_{h}\right)-\left(\mathcal{L}\left(\boldsymbol{w}_{h}\right), \Pi_{h} \operatorname{curl} A \boldsymbol{u}\right)+b\left(\boldsymbol{w}_{h}, p\right)-\left(\boldsymbol{u}, \boldsymbol{w}_{h}\right) .
$$

Elementwise integration by parts leads to

$$
\begin{aligned}
r_{h}\left(\boldsymbol{w}_{h}\right)= & \sum_{T \in \mathcal{T}_{h}} \int_{T} \operatorname{curl} \operatorname{curl} A \boldsymbol{u} \cdot \boldsymbol{w}_{h}+\int_{\mathcal{E}} \underline{\left[\boldsymbol{w}_{h}\right]_{T}} \cdot\{\{\operatorname{curl} A \boldsymbol{u}\}\} \\
& -\left(\mathcal{L}\left(\boldsymbol{w}_{h}\right), \Pi_{h} \operatorname{curl} A \boldsymbol{u}\right)+b\left(\boldsymbol{w}_{h}, p\right)-\left(\boldsymbol{u}, \boldsymbol{w}_{h}\right) .
\end{aligned}
$$

As (28) implies that curl curl $A \boldsymbol{u}+\nabla p=\boldsymbol{u}$, we finally arrive at

$$
r_{h}\left(\boldsymbol{w}_{h}\right)=\int_{\mathcal{E}} \underline{\left.\left[\boldsymbol{w}_{h}\right]\right]_{T}} \cdot\left\{\left\{\operatorname{curl} A \boldsymbol{u}-\Pi_{h} \operatorname{curl} A \boldsymbol{u}\right\}\right\} .
$$

Cauchy-Schwarz's inequality and Lemma 4.3 of [19] yield

$$
\begin{aligned}
\left|r_{h}\left(\boldsymbol{w}_{h}\right)\right| & \leq \sum_{E \in \mathcal{E}}\left\|\underline{\llbracket \boldsymbol{w}_{h} \rrbracket_{T}}\right\|_{E}\left\|\left\{\left\{\operatorname{curl} A \boldsymbol{u}-\Pi_{h} \operatorname{curl} A \boldsymbol{u}\right\}\right\}\right\|_{E} \\
& \leq \mathrm{C} \sum_{T \in \mathcal{T}_{h}} h_{T}^{t-1 / 2}\|\operatorname{curl} A \boldsymbol{u}\|_{t, T} \sum_{E \in \mathcal{E}, E \subset T}\left\|\underline{\left.\llbracket \boldsymbol{w}_{h}\right]_{T}}\right\|_{E},
\end{aligned}
$$


for any $t \in] 1 / 2,1]$. Using discrete Cauchy-Schwarz's inequality and the definition of the norm $\|\cdot\|_{D G, h}$, we obtain

$$
\left|r_{h}\left(\boldsymbol{w}_{h}\right)\right| \leq \mathrm{C} h^{t}\|\operatorname{curl} A \boldsymbol{u}\|_{t, \Omega}\left\|\boldsymbol{w}_{h}\right\|_{D G, h} .
$$

The theorem will be proved if one can show that curl $A \boldsymbol{u}$ belongs to $H^{t}(\Omega)$ for some $t>1 / 2$. As $A \boldsymbol{u}$ belongs to $X$ and curl curl $A \boldsymbol{u}=\boldsymbol{u}-\nabla p$ belongs to $L^{2}(\Omega)^{3}$, we see that

$$
\operatorname{curl} A \boldsymbol{u} \in X_{T}(\Omega),
$$

where $X_{T}(\Omega)$ is defined by (see [13])

$$
X_{T}(\Omega):=\left\{\boldsymbol{v} \in L^{2}(\Omega)^{3}: \operatorname{curl} \boldsymbol{v} \in L^{2}(\Omega)^{3}, \operatorname{div} \boldsymbol{v} \in L^{2}(\Omega) \text { and } \boldsymbol{v} \cdot \boldsymbol{n}=0 \text { on } \Gamma\right\} .
$$

The conclusion follows from the well known embedding $X_{T}(\Omega) \hookrightarrow H^{t}(\Omega)$ for some $t>1 / 2$, consequence of Theorem 1.1 from [13] for instance (see also $[5,6]$ ).

Corollary 6.5. Let $\lambda$ be a positive eigenvalue of the continuous problem (4) of multiplicity $m$. Then for $h$ small enough the discrete problem (7) has exactly $m$ positive eigenvalues $\lambda_{h, j}, j=1, \ldots, m$ (repeated according to their multiplicity) such that

$$
\lambda_{h, j} \rightarrow \lambda \text { as } h \rightarrow 0, \forall j=1, \ldots, m .
$$

Proof. We have verified in Theorems 6.3 and 6.4 the assumptions of Theorem 6.2 for the set $\mathcal{A}$ introduced in Theorem 6.3. Denote by $\mu=\frac{1}{\lambda}$ the corresponding eigenvalue of $A$ (see Sect. 2). By Theorem 6.2, for $h$ small enough, $A_{h}$ has exactly $m$ positive eigenvalues $\mu_{h, j}, j=1, \ldots, m$, moreover the estimate (25) implies

$$
\left|\mu-\mu_{h, j}\right| \leq \mathrm{C}\left(\sum_{j=1}^{m}\left\|\left(A-A_{h}\right) \phi_{j}\right\|_{X}^{2}\right)^{1 / 2} .
$$

By the pointwise convergence of $A_{h}$ to $A$, we deduce that

$$
\mu_{h, j} \rightarrow \mu \text { as } h \rightarrow 0, \forall j=1, \ldots, m,
$$

and we conclude by Lemma 5.3 .

\section{Some Numerical TESTS}

For the sake of simplicity, our theoretical results are tested on two-dimensional domains. In fact using the results from [15,24], our theoretical results also hold for two-dimensional domains, meshed by a regular family (in Ciarlet's sense) of triangles. For our tests we take $k=1$ and compute the first fifth eigenvalues for the square domain $(0,1)^{2}$ and the $L$-shape domain $(-1,1)^{2} \backslash(0,1)^{2}$, as well as the first four eigenvalues for the sector domain given in polar coordinates by $\left\{(r, \theta) \in \mathbb{R}^{2} ; 0 \leq r \leq 1,0 \leq \theta \leq \frac{3 \pi}{2}\right\}$. For the first domain, analytical solutions are known (see for instance [9]) and the non zero eigenvalues of problem (1) are given by

$$
\lambda_{j, l}=\left(j^{2}+l^{2}\right) \pi^{2}, j+l \geq 1,
$$

in other words, for $j \geq 1$, the number $j^{2} \pi^{2}$ is a double eigenvalue, while for $j, l \geq 1, j \neq l$, the number $\left(j^{2}+l^{2}\right) \pi^{2}$ is a simple eigenvalue. For the second domain, no analytical solution exists but we compare our results with the computational eigenvalues presented by the benchmark of M. Dauge [14] obtained by using a Galerkin approximation of the Neumann eigenvalues with a geometrical refined mesh near the corner and polynomials of high degree. For the third domain, analytical solutions are known (see for instance [15]). 
Since the discrete problem (7) has a quite large kernel and therefore does not correspond to an eigenvalue problem for positive definite matrices we use the discrete regularization technique (compare with [3,18]): first we remark that the kernel $K_{h}$ of problem (7) is explicitly known, namely

$$
K_{h}=\nabla S_{h}
$$

Taking into account Lemma 5.2 we further remark that $\lambda_{h}$ is a nonzero eigenvalue of (7) if and only if there exists $\boldsymbol{E}_{h} \in X_{h}$ solution of

$$
a_{h}\left(\boldsymbol{E}_{h}, \boldsymbol{v}_{h}\right)=\lambda_{h}\left(\boldsymbol{E}_{h}, \boldsymbol{v}_{h}\right), \forall \boldsymbol{v}_{h} \in V_{h} .
$$

Now we fix a basis $\left\{\lambda_{j}\right\}_{j=1}^{J}$ of $S_{h}$ and a basis $\left\{\boldsymbol{v}_{i}\right\}_{i=1}^{I}$ of $V_{h}$ (clearly $J<I$, but $J$ is quite large). Writing

$$
\boldsymbol{E}_{h}=\sum_{i=1}^{I} x_{i} \boldsymbol{v}_{i},
$$

we see that the eigenvalue problem (29) is equivalent to

$$
A X=\lambda_{h} B X,
$$

where $X=\left(x_{i}\right)_{i=1}^{I}$ is the unknown vector, $A=\left(a\left(\boldsymbol{v}_{i}, \boldsymbol{v}_{i^{\prime}}\right)\right)_{1 \leq i, i^{\prime} \leq I}$ is the "rigidity" matrix and $B=\left(\left(\boldsymbol{v}_{i}, \boldsymbol{v}_{i^{\prime}}\right)\right)_{1 \leq i, i^{\prime} \leq I}$ is the mass matrix. Using the basis of $S_{h}$ we can say that $\boldsymbol{E}_{h}$ belongs to $X_{h}$ if and only if

$$
C^{\top} X=0
$$

where $C=\left(c_{i j}\right)_{1 \leq i \leq I, 1 \leq j \leq J}$, with $c_{i j}=\left(\boldsymbol{v}_{i}, \nabla \lambda_{j}\right)$.

In summary the eigenvalue problem is reduced to find $X$ satisfying (31) and solution of (30).

At this stage taking an arbitrary weight $s>0$, we see that $X$ satisfying (31) and solution of (30) also satisfies

$$
\left(A+s C C^{\top}\right) X=\lambda_{h} B X .
$$

The main point is that $A+s C C^{\top}$ is a positive definite matrix. Indeed we clearly have

$$
X^{\top}\left(A+s C C^{\top}\right) X \geq 0, \forall X \in \mathbb{R}^{I} .
$$

On the other hand if

then

$$
X^{\top}\left(A+s C C^{\top}\right) X=0,
$$

$$
X^{\top} A X=0 \text { and }\left\|C^{\top} X\right\|=0,
$$

and consequently $X$ belongs to the kernel of $A$ and to its orthogonal, so that $X=0$.

The second advantage of the eigenvalue problem (32) is that the zero eigenvalues of problem (30) become positive but depend linearly on $s$ (while the non zero eigenvalues of problem (30) are unchanged). Consequently the nonzero eigenvalues of interest can be captured by choosing the parameter $s$ large enough (or by taking different values of $s$ ). In that respect, our method is similar to the discrete regularization method for standard edge elements $[3,18]$ and can be compared with the so-called grad-div regularizated method for the continuous Maxwell's problem where the term $s \int_{\Omega} \operatorname{div} \mathbf{u} \operatorname{div} \mathbf{v}$ is added to the standard bilinear form of the curl $[13,16]$.

The approximated eigenvalues are then produced by solving the problem (32) using Matlab; the matrices being generated by our finite element code Simula + . For both problems we have fixed the penalty parameter $\alpha$ equal to 100 and the weight $s=3200$ in order that the first eigenvalues are the expected ones.

In Figure 1 we have plotted the first fifth eigenvalues for the square in comparison with the exact ones for different meshes, while in Figure 2 the error is given as a function of the DoF. This last figure demonstrates the convergence of the discrete eigenvalues to the exact ones with a convergence rate of 1 in $D o F^{-1}$ 


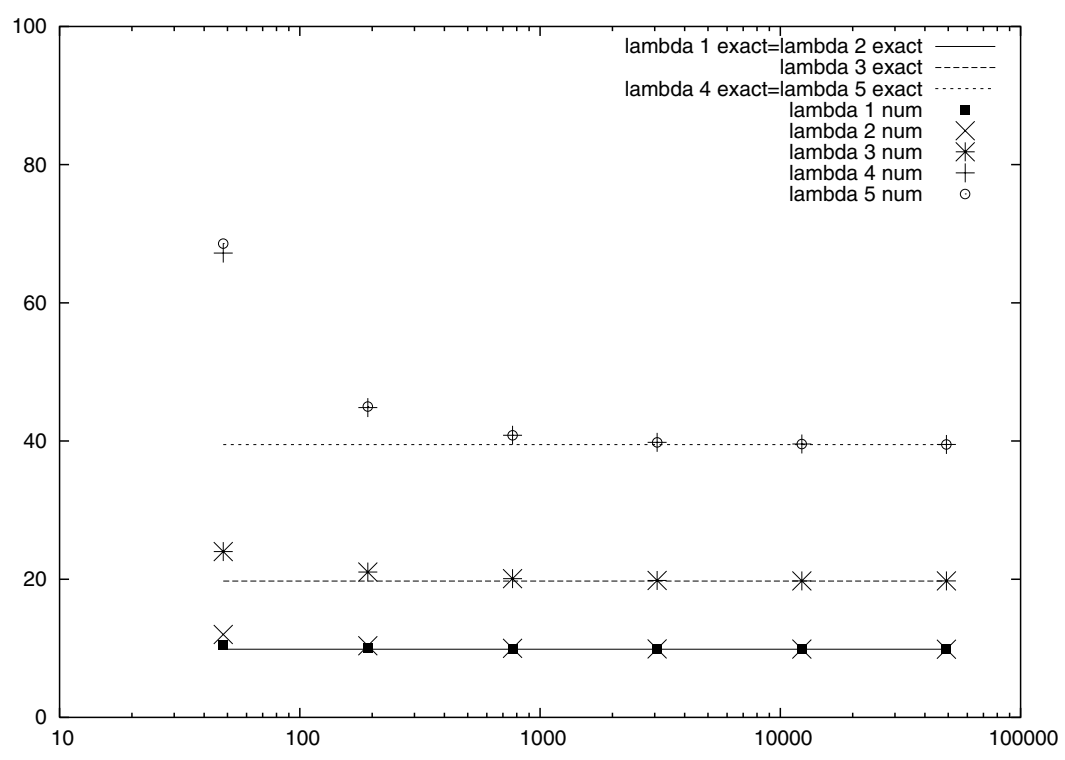

Figure 1. Eigenvalues wrt DoF for the unit square domain.

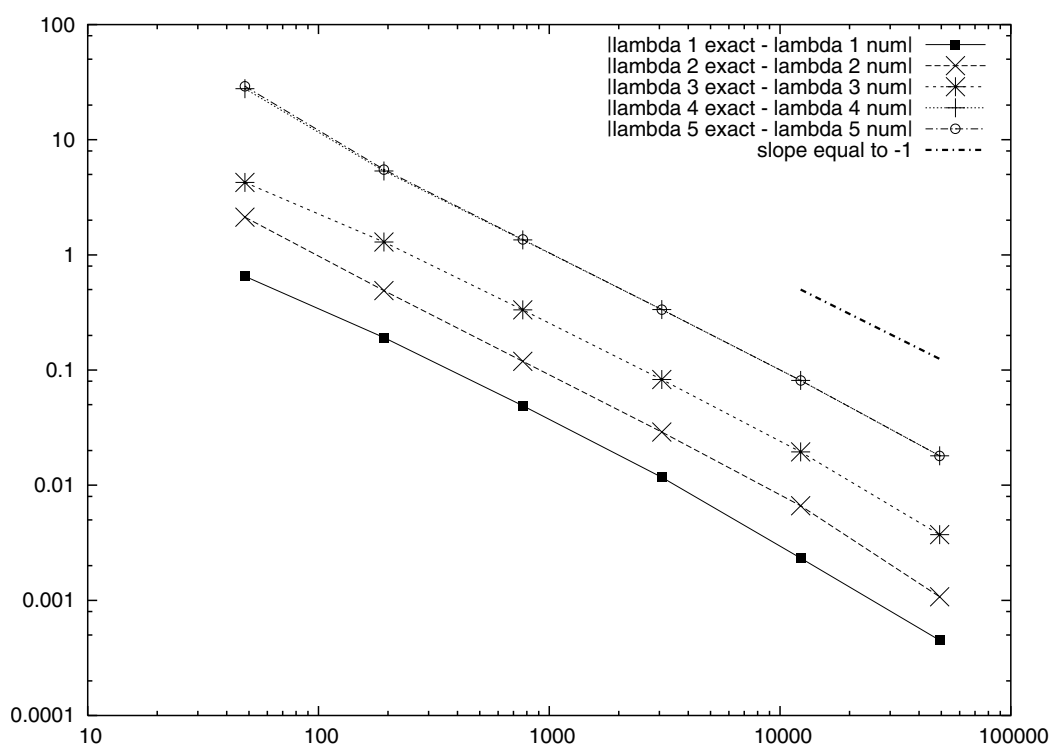

FiguRE 2. Convergence rates in $D o F$ for the unit square domain.

(i.e. of order 2 in $h$ ). Figures 3 and 4 present the same results for the $L$-shape domain, by taking as exact values the one given by M. Dauge [14]. Here again, a convergence rate of order 1 in $D o F^{-1}$ (or of order 2 in $h$ ) is obtained. We should only notice the particular behaviour of the first eigenvalue convergence rate: the graph of the error for this smallest eigenvalue exhibits a sudden rise after a dramatic fall, and the convergence rate is no more equal to 1 with respect to $D o F^{-1}$. This phenomenon occurs for another domain (the slit domain) in [15], for which the smallest eigenvalue also corresponds to a singular eigenfield. Moreover, also for the $L$-shape, numerical results obtained in [17] confirm that there exists a strong correlation between the convergence rate and the regularity of the corresponding eigenfunction. In Figure 5 we have plotted the first four eigenvalues for 


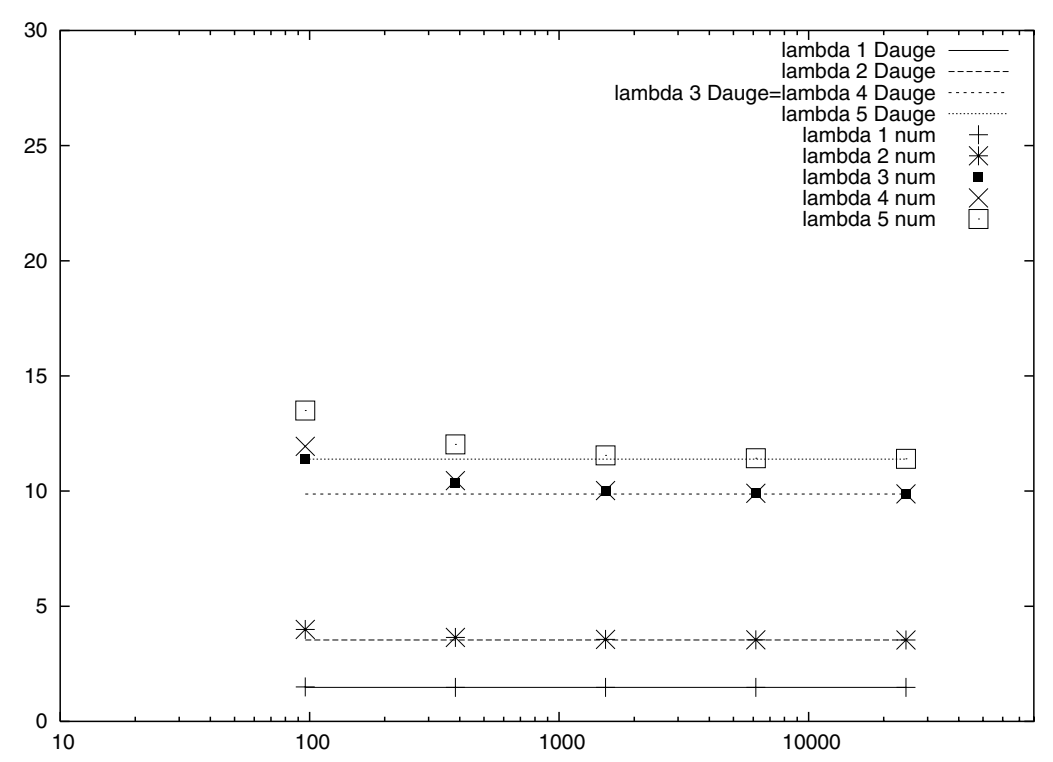

Figure 3. Eigenvalues wrt $D o F$ for the $L$-shape domain.

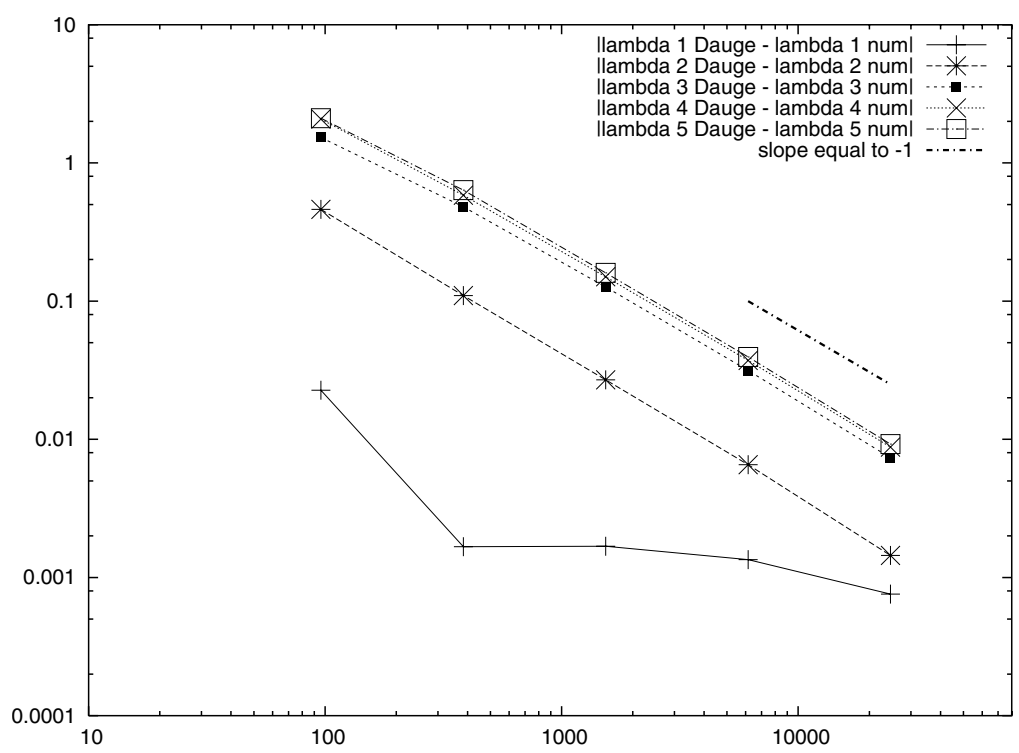

FIgURE 4. Convergence rates in $D o F$ for the $L$-shape domain.

the sector domain in comparison with the exact ones for different meshes, while in Figure 6 the error is given as a function of the $D o F$. For this last example, the convergence properties are the same as the ones obtained for the $L$-shape domain. Note further that these results are comparable with those from [15].

Acknowledgements. The tests presented in this section have been performed with the help of the SIMULA+ finite element code (MACS, University of Valenciennes and LPMM, University and ENSAM of Metz, both in France). 


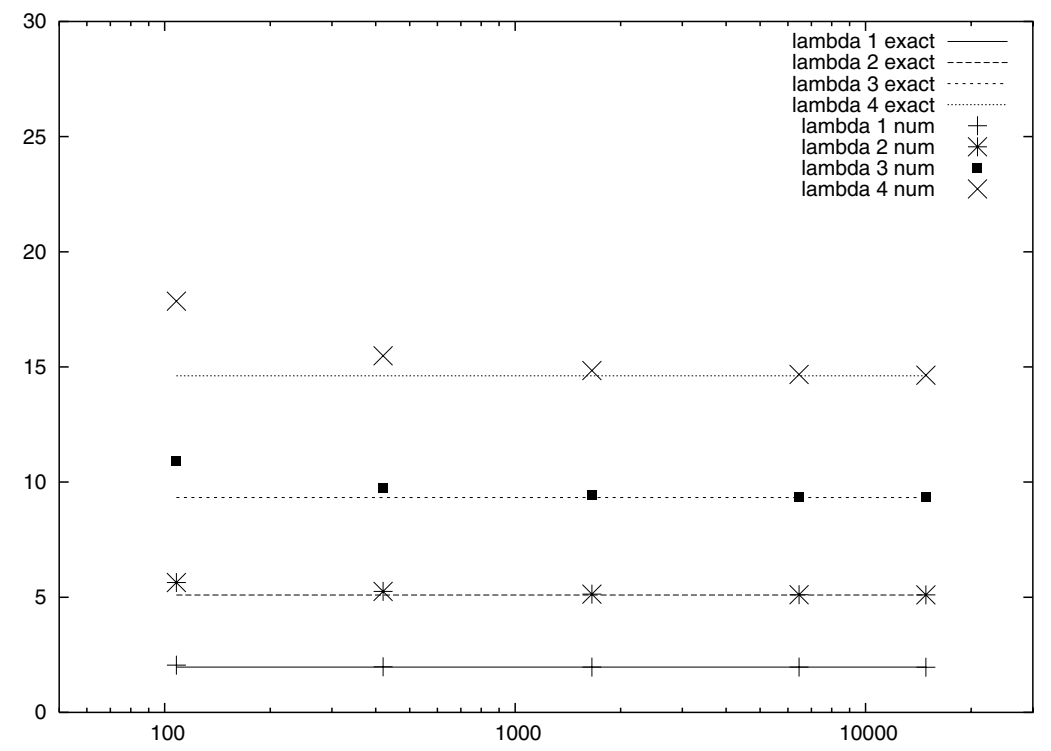

FiguRE 5. Eigenvalues wrt $D o F$ for the sector domain.

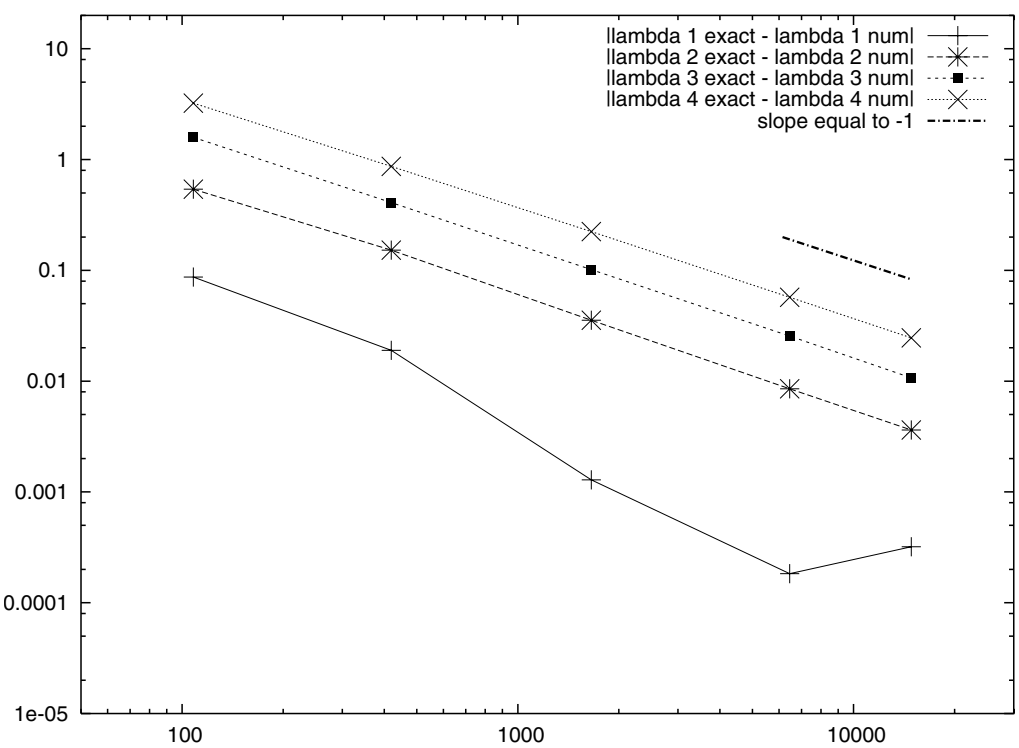

FiguRE 6. Convergence rates in $D o F$ for the sector domain.

\section{REFERENCES}

[1] P. Anselone, Collectively compact operator approximation theory. Prentice Hall (1971).

[2] T. Apel and S. Nicaise, The finite element method with anisotropic mesh grading for elliptic problems in domains with corners and edges. Math. Method. Appl. Sci. 21 (1998) 519-549.

[3] P. Arbenz and R. Geus, Eigenvalue solvers for electromagnetic fields in cavities, in High performance scientific and engineering computing, H.-J. Bungartz, F. Durst, and C. Zenger, Eds., Lect. Notes Comput. Sc., Springer, Berlin 8(1999).

[4] D.G. Arnold, F. Brezzi, B. Cockburn and L.D. Marini, Unified analysis of discontinuous Galerkin methods for elliptic problems. SIAM J. Numer. Anal. 39 (2001) 1749-1779. 
[5] F. Assous, P. Ciarlet and E. Sonnendrücker, Characterization of the singular part of the solution of Maxwell's equations in a polyhedral domain. RAIRO Modél. Math. Anal. Numér. 32 (1998) 485-499.

[6] M. Birman and M. Solomyak, $L^{2}$-theory of the Maxwell operator in arbitrary domains. Russ. Math. Surv. 42 (1987) 75-96.

[7] D. Boffi, Fortin operator and discrete compactness for edge elements. Numer. Math. 87 (2000) 229-246.

[8] D. Boffi, L. Demkowicz and M. Costabel, Discrete compactness for $p$ and $h p 2$ d edge finite elements. Math. Mod. Meth. Appl. S. 13 (2003) 1673-1687.

[9] D. Boffi, M. Costabel, M. Dauge and L. Demkowicz, Discrete compactness for the hp version of rectangular edge finite elements. ICES Report 04-29, University of Texas, Austin (2004).

[10] F. Brezzi and M. Fortin, Mixed and hybrid finite element methods. Springer, New York (1991).

[11] F. Chatelin, Spectral approximation of linear operators. Academic Press, New York (1983).

[12] P.G. Ciarlet, The finite element method for elliptic problems. North-Holland, Amsterdam (1978).

[13] M. Costabel and M. Dauge, Singularities of electromagnetic fields in polyhedral domains. Arch. Rational Mech. Anal. 151 (2000) 221-276.

[14] M. Dauge, Benchmark computations for Maxwell equations for the approximation of highly singular solutions. Technical report, University of Rennes 1. http://perso.univ-rennes1.fr/monique.dauge/core/index.html

[15] L. Demkowicz, P. Monk, C. Schwab and L. Vardepetyan, Maxwell eigenvalues and discrete compactness in two dimensions. Comput. Math. Appl. 40 (2000) 589-605.

[16] C. Hazard and M. Lenoir, On the solution of time-harmonic scattering problems for Maxwell's equations. SIAM J. Math. Anal. 27 (1996) 1597-1630.

[17] J. Hesthaven and T. Warburton, High-order nodal discontinuous Galerkin methods for the Maxwell eigenvalue problem. Philos. T. Roy. Soc. A 362 (2004) 493-524.

[18] R. Hiptmair, Finite elements in computational electromagnetism. Acta Numer. 11 (2002) 237-339.

[19] P. Houston, I. Perugia, D. Schneebeli and D. Schötzau, Interior penalty method for the indefinite time-harmonic Maxwell equations. Numer. Math. 100 (2005) 485-518.

[20] P. Houston, I. Perugia and D. Schötzau, Mixed discontinuous Galerkin approximation of the Maxwell operator: Non-stabilized formulation. J. Sci. Comput. 22 (2005) 315-346.

[21] F. Kikuchi, On a discrete compactness property for the Nédélec finite elements. J. Fac. Sci. U. Tokyo IA 36 (1989) $479-490$.

[22] M. Krizek and P. Neittaanmaki, On the validity of Friedrichs' inequalities. Math. Scand. 54 (1984) 17-26.

[23] R. Leis, Initial boundary value problems in Mathematical Physics. John Wiley, New York (1988).

[24] S. Lohrengel and S. Nicaise, A discontinuous Galerkin method on refined meshes for the 2d time-harmonic Maxwell equations in composite materials. Preprint Macs, University of Valenciennes, 2004. J. Comput. Appl. Math. (to appear).

[25] P. Monk, Finite element methods for Maxwell's equations. Numer. Math. Scientific Comp., Oxford Univ. Press, New York (2003).

[26] P. Monk and L. Demkowicz, Discrete compactness and the approximation of Maxwell's equations in $\mathbb{R}^{3}$. Math. Comp. 70 (2000) 507-523.

[27] J. Osborn, Spectral approximation for compact operators. Math. Comp. 29 (1975) 712-725. 